\title{
Why COVID-19 trials should be blinded (as any other one)
}

\section{Rafael Leite Pacheco ${ }^{1}$ (1) Ana Luiza Cabrera Martimbianco ${ }^{2}$ (1) Carolina de Oliveira Cruz Latorraca ${ }^{3}$ (1) Rachel Riera ${ }^{4}$ (1)}

\begin{abstract}
${ }^{1}$ Corresponding author. Universidade Federal de São Paulo (São Paulo), Centro Universitário São Camilo (São Paulo). São Paulo, Brazil. rleitepacheco@hotmail.com ${ }^{2}$ Centro Universitários São Camilo (São Paulo), Universidade Metropolitana de Santos (Santos). São Paulo, Brazil. analuizacabrera@hotmail.com 3Universidade Federal de São Paulo (São Paulo). São Paulo, Brazil. caru.pepm@yahoo.com.br ${ }^{4}$ Núcleo de Avaliação de Tecnologias em Saúde, Hospital Sírio-Libanês (São Paulo); Universidade Federal de São Paulo (São Paulo).
\end{abstract} São Paulo, Brazil. rachelriera@hotmail.com

KEYWORDS: Blinding. Clinical trials. COVID-19.

\section{Main text}

The COVID-19 pandemic led to an enormous research effort and collaboration worldwide. To this date, hundreds of clinical trials are being developed assessing interventions to prevent and treat the COVID-19 infection and complications ${ }^{1}$.

Some of these clinical trials are assessing intubated and/or intensive care unit patients. Because of this, some trials are not using placebos, under the argument that this population will not be influenced by the knowledge of intervention assignment.

Despite patients are an important unit that should be blinded in a clinical trial, there are several benefits in blinding the clinical trial staff (performance bias) and the outcome assessors (detection bias). These estimates vary substantially in different studies, but previous reports showed that high risk of detection bias may increase the estimative of the effects in $36 \%$ and an average of 13 to $15 \%$ on high risk performance bias trials ${ }^{23}$.

The lack of blinding is always more worrisome when subjective outcomes or patient reported outcomes are being assessed 3 . However, the lack of blinding can bring avoidable bias even on studies assessing 'hard outcomes', such as mortality, where patients knowledge of enrollment group is irrelevant. We will give three justifications why you should blind your staff and outcome assessors on COVID-19 trials:

\section{Non-protocol co-interventions may be imbalanced in an open label trial}

When the clinical staff are aware of the patient's assignment group, there may be unconscious or conscious differences in the clinical treatment and support given between groups. This is very worrisome, as these differences may be the cause of any observed outcome effect.

This situation was observed in several published clinical trials. An example is a clinical trial that 
was published in 2012 in The Lancet ${ }^{4}$, comparing minimally invasive surgery versus open surgery for treating patients with esophageal cancer. The patients on the minimally invasive surgery received different type of lung-ventilation and a disproportional scheme of anesthesia. The imbalances in these two co-interventions might had led to distortion on the observed outcome estimates.

In the first open-label non-randomized controlled trial assessing hydroxychloroquine for patients with COVID-19 we already found an imbalance in the cointerventions administered between groups ${ }^{5}$. In the hydroxychloroquine arm, 30\% (6/20) of the patients also received azithromycin in comparison of $0 \%$ $(0 / 16)$ in the control group. Although it's hard to assess the impact of this imbalance on the outcome assessment in this case, this can indicate that other co-interventions were also imbalanced.

\section{Violations in the randomization protocol can occur more often in an open label trial}

In a multi-center randomized controlled trial assessing prehospital antibiotics in the ambulance for sepsis ${ }^{6}$, the authors reported a major protocol violation that would have been avoided if the studied was blinded. It was reported that more patients were included in the intervention group, with the probable reason being 'the overenthusiasm of EMS personnel wanting to treat as many patients as possible with antibiotics in the ambulance as this was an open-label study. To achieve this, some EMS personnel purposefully opened the envelopes until they found an envelope instructing randomization to the intervention group'. In the end of the trial, the imbalance on the number of patients were huge: 1150 on the control group compared to 1548 on the antibiotics group.

In the COVID-19 scenario, this may be very important. The hospitals are operating with maximum capacity and usually understaffed. The clinical trials are being developed fast, and the training of the clinical staff is being shortened. So, it's expected that protocol violations as this can occur, mainly including patients with worse prognosis in the intervention group.

\section{Non-planned interim analysis are dangerous and may occur frequently in an open label trial}

The importance of pre-planning the outcomes and time-points are well established and recognized in the literature. Interim analysis may be useful if they are pre-planned and used with caution.

In a clinical trial where the outcome assessors and main investigators are constantly being updated on the status of the patients, non-planned interim analysis may be misleading.

Let's discuss the above mentioned first trial that assessed hydroxychloroquine for COVID-195. The authors state in the discussion: 'For ethical reasons and because our first results are so significant and evident we decide to share our findings with the medical community, given the urgent need for an effective drug against SARS-CoV-2 in the current pandemic context'. The primary outcome was reported at 6-days of follow-up. This time point was not planned in the study protocol. The longest followup planned was 14 days.

The authors based their conclusions on the fact that at day six, 70\% (14/20) of the patients in the hydroxychloroquine had a negative viral detection in comparison of $12.5 \%(2 / 16)$ of the patients in the control group. This is an expressive difference, but what if at day $8,90 \%$ of the patients in both groups become negative? Would the 'solid conclusion' of effect be the same? Probably not.

Although it is impossible to know what will happen on day 14 of the follow-up until results are fully published, the fact that authors enthusiastically concluded effectiveness on day six may had led to precipitate recommendations for the use of this intervention on COVID-19 patients.

Concluding, we presented three arguments to justify the blinding of clinical trials even in scenarios where the assignment knowledge by the patients are impossible or irrelevant. We expect that blinding in COVID-19 trials will only moderate increase the complexity and will have very low impact on the cost of the study. Considering the all the avoidable biases, blinding should always be performed if possible. 


\section{Author contributions}

All authors participated in the manuscript conception, writing and approving of the final draft.

\section{Competing interests}

No financial, legal or political competing interests with third parties (government, commercial, private foundation, etc.) were disclosed for any aspect of the submitted work (including but not limited to grants, data monitoring board, study design, manuscript preparation, statistical analysis, etc.).

\section{References}

1. Aronson J, Ferner R, DeVito NJ, Heneghan C. COVID-19 Registered Trials - and analysis. CEBM. [Internet]. 2020. [acesso em 2020 mar. 27] Disponível em: https://www.cebm.net/ covid-19/registered-trials-and-analysis/
2. Catalogue of Bias Collabaration, O'Sullivan J, Banerjee A, Pluddemann A. Detection bias. [Internet]. 2017. [acesso em 2020 mar. 27]. Disponível em: https://catalogofbias.org/biases/ detection-bias/

3. Catalogue of Bias Collaboration, Banerjee A, Pluddemann A, O'Sullivan J, Nunan D. Performance bias. [Internet]. 2019. [acesso em 2020 mar. 27]. Disponível em: https://catalogofbias/biases/ performance-bias/

4. Biere SS, van Berge HM, Maas KW, Bonavina L, Rosman C, Garcia JR et al. Minimally invasive versus open oesophagectomy for patients with oesophageal cancer: a multicentre, open-label, randomised controlled trial. Lancet. 2012;379(9829):1887-92. doi: 10.1016/S0140-6736(12)60516-9

5. Gautret P, Lagiera JC, Parola P, HagVno T, Hoang VT, Meddeb $L$ et al. Hydroxychloroquine and azithromycin as a treatment of COVID-19: results of an open-label non-randomized clinical trial. International Journal of Antimicrobial Agents. 2020. doi: 10.1016/j. ijantimicag.2020.105949

6. Alam N, Oskam E, Stassen PM, Exter PV, van de Ven PM, Haak HR et al. Prehospital antibiotics in the ambulance for sepsis: a multicentre, open label, randomised trial. Lancet Respir Med. 2018;6(1):40-50. doi: 10.1016/S2213-2600(17)30469-1 